\title{
An Adaptive and Distributed Traffic Management System for Vehicular ad-hoc Networks
}

\author{
Thiago S. Gomides ${ }^{1}$ (author) and Daniel L. Guidoni ${ }^{1}$ (advisor) \\ ${ }^{1}$ Departamento de Ciência da Computação \\ Universidade Federal de São João Del Rei, (UFSJ) - MG - Brasil \\ \{gomides, guidoni\}@ufsj.edu.br
}

\begin{abstract}
The economic and environmental impacts caused by traffic congestion have become a cause for concern in urban centers. Improvements in the city's road infrastructure for minimizing these impacts are expensive and take time to be executed. In this scenario, Traffic Management Systems (TMSs) emerge as efficient and technological alternatives to tackle vehicular traffic issues. This work proposes a TMS based on vehicular communication to improve vehicular traffic flow in dense urban centers. The proposed work aims to study the following TMS issues: (i) how to estimate traffic jams; (ii) propose proactive and reactive information sharing protocols; (iii) propose an adaptive traffic sharing information that has less impact on the network with more information available and (iv) real-time and periodical new route estimation. The performance evaluation shows our solutions' ability to minimize traffic jams and improve mobility, with a low communication overhead compared to state-of-the-art solutions.
\end{abstract}

\section{Problem characterization and Motivation}

The next generation of Smart Cities will rely heavily on Intelligent Transport Systems (ITSs) due to the constant increase in the complexity and dynamism of traffic caused by continued urbanization and population growth [Cookson and Pishue 2020]. Dealing with overcrowded cities is challenging mainly due to the lack of adequate investments in urban infrastructure and services [Madlener and Sunak 2011]. In addition, the impacts of poor governance on urban management intensify and increase the emergence of traffic problems, such as traffic jams and vehicular accidents that affect people's lives, environment, economy, and society [Ekblad 1993].

In such a scenario, Traffic Management Systems (TMSs) play a crucial role in supporting the design and development of innovative, resilient, smart, and sustainable systems with a lower cost compared to maintenance/increase of the road infrastructure. As a result of the automotive industry's rapid development, ITS solutions have become even more popular worldwide, boosted mainly by using Vehicular Ad Hoc Networks (VANETs). Other technologies such as the 5G mobile networks, the development of autonomous and connected vehicles, urban computing, and the Internet of Things (IoT) [Zhou et al. 2020] also substantially influenced ITS's popularity.

Understanding the challenges and characteristics of the vehicular networks is a crucial concern in developing an efficient TMS. Although VANETs share some characteristics with the traditional mobile wireless and ad hoc networks, such as frequent network topology changes, the nodes' organization is more dynamic in the VANETs scenario. Exploring the organization of nodes in VANETs by understanding the characteristics of 
each communication subset - Vehicle-to-Vehicle (V2V), Vehicle-to-Infrastructure (V2I), Vehicle-to-Everything (V2X), and Hybrid - and their impact on the overall system performance is the first key challenge faced in TMS development. Due to the high mobility behavior of vehicles using $\mathrm{V} 2 \mathrm{~V}$ communication, frequent communication disconnections can occur. Thus, a proper data communication protocol is essential for a distributed TMS execution.

\section{Objectives and Contributions}

The contributions of the thesis advance the state-of-the-art by proposing collaborative, adaptive, and distributed TMS's algorithms and protocols for traffic management in vehicular ad-hoc networks.

The main objective of this thesis is to propose a distributed TMS algorithms and protocols in vehicular ad-hoc networks. The use of only inter-vehicle communication requires collaboration among the nodes in collecting and sharing the information. In a V2V-TMS environment, the nodes should make traffic information available to manage traffic jams and increase traffic flow in urban centers. Figure 1 presents the TMS framework that guided this thesis development and, as specific objectives, we investigate and propose solutions for the Displacement Analysis, Information Sharing, and Route Decision modules. The Displacement Analysis enables the local assessment of congestion on the streets/roads where vehicles travel. The Information Sharing is responsible for data communication, where each vehicle verifies if the estimated level of congestion should be disseminated to its neighbors. The Route Decision is responsible for estimating alternative routes using local information received from other vehicles.

Each vehicle in our VANET environment can process, analyze, communicate, and make distributed decisions. The vehicles are equipped with: On-Board Unit (OBU), IEEE 802.11p communication interface, Global Position System (GPS) receiver, and the road map with the city characteristics, including roads segment definitions, such as dimensions, number of lanes, traffic lights positions, and maximum allowed speed. The contributions of the thesis advance the state-of-the-art by proposing collaborative, adaptive, and distributed solutions, which are presented in the following.

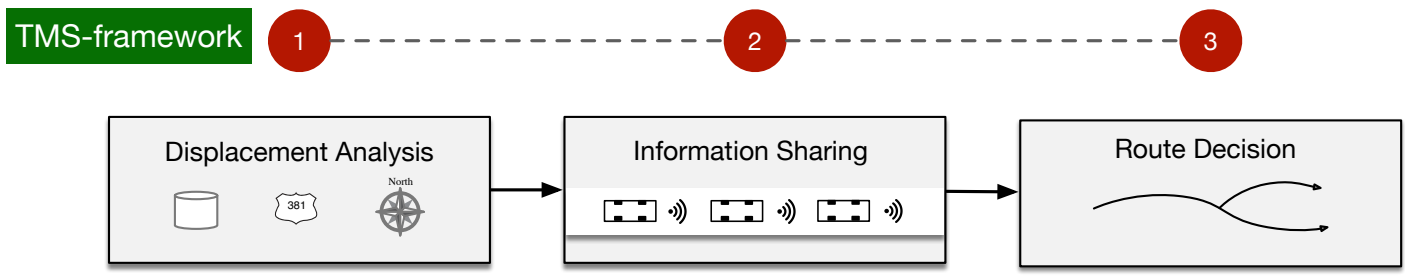

Figure 1. TMS framework

Contribution 1 - Solutions for the Displacement Analysis. In the literature, the techniques for detecting and classifying traffic jams are based on occupancy or travel time on roads. The road occupancy method considers the number of vehicles traveling on the road and requires cooperation or a centralized information-sharing system; however, road travel time is possible to be measured by each vehicle. For the cooperative strategy, we propose a solution where all vehicles on the road disseminate a periodical hello 
message for the road occupancy estimation. Based on the estimated value, the vehicle is able to estimate traffic jams using traffic engineering concepts and the city map features. Considering only local information, we propose a travel distance analysis classification presented in Figure 1. During its route, each vehicle evaluates its displacement in terms of (i) Traveled Distance (TD) considering the road segment the vehicle travels and the related time spent to achieve the spatial progress and (ii) Expected Distance (ED) that the vehicle should travel if the road has a free-flow condition. We introduce $\Delta$ ED to represent a range of free-flow conditions to consider different driver' behaviors. Considering the distance $\phi$, the vehicle can estimate the congestion level it is facing while moving on the considered road. As soon as $\phi$ increases, the vehicle is observing traffic jams.

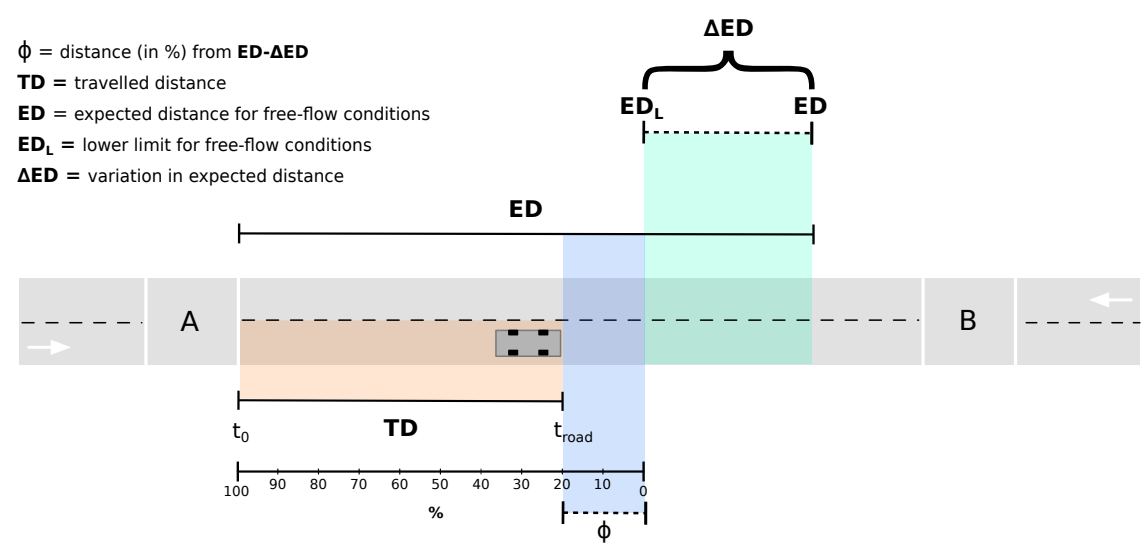

Figure 2. Displacement analysis within a road segment

Comparing both proposed solutions, cooperative traffic flow classification demands traffic information from multiple vehicles on the same road to create a traffic consensus, increasing traffic messages but with a high precision traffic estimation. However, our analyses showed that the cooperative classification gain is not substantial to justify the number of exchanged messages to create it. We adopt the local travel distance displacement analysis in the proposed TMS solution.

Contribution 2 - Solutions for Information Sharing. The data sharing protocols should rely upon vehicle-to-vehicle communication, and the vehicles should share their estimated traffic classification. In the thesis, we investigated different strategies for data sharing, including single-hop and multi-hop communication. One-hop information sharing is used to create a local and distributed knowledge of the traffic conditions with a low number of messages. As the solution uses only the traffic information regarding the next road decision, the information sharing procedure transmits the message only for its one-hopstreet neighborhood, i.e., only vehicles on one-hop-streets receive the traffic information message. When the vehicle faces a next road decision (go forward or turn), the received information is used to calculate the next conversion based on the congestion level of the next streets. If the estimated traffic flow is low, a single-hop communication is sufficient to notify one-hop-ahead vehicles in order to find alternative routes. Figure 3(a) illustrates the single-hop communication strategy, where the vehicle moving from point A to point $\mathrm{F}$ follows a sequence of three next-road decisions. The green path represents the shortest distance, and the orange one represents the fastest (considering the received traffic information). 
When the vehicle is observing a severe traffic jam, we consider that nearby roads also have the same issue, and multi-hop communication should notify more vehicles. Figure 3(b) illustrates the multi-hop communication, and our findings suggest that the multi-hop communication should be adaptive considering the traffic jam observation. For small values of $\phi$, one-hop information sharing is sufficient and, as $\phi$ increases, the multihop can be 2/3/4-hop information sharing. For severe traffic jams, four-hop information sharing takes place.

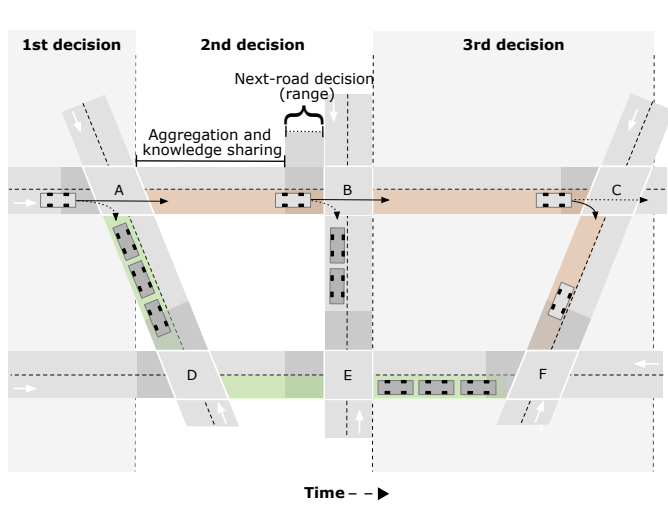

(a) Single-hop

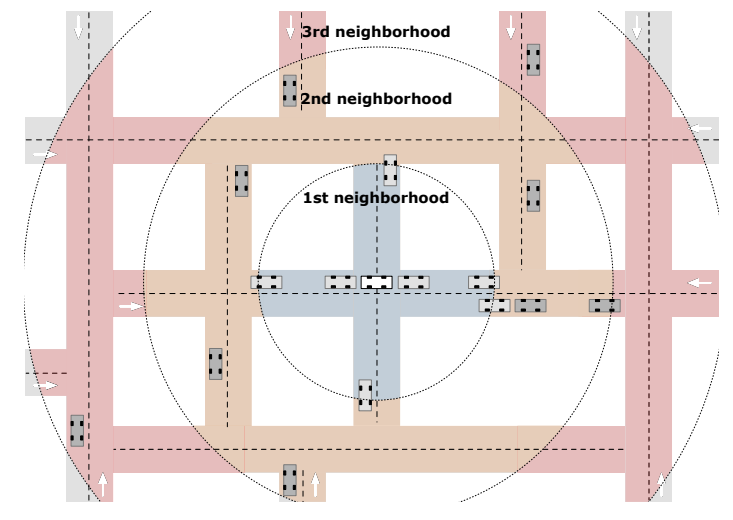

(b) Multi-hop

Figure 3. Sigle-hop and multi-hop communication strategies.

Besides single-hop or multi-hop communication, we develop proactive and reactive traffic sharing information protocols, and Figure 4 shows the overview of the information sharing. After the road traffic analysis, the Proactive information sharing ensures high availability of information since the vehicle always shares its estimated road traffic condition. The information-sharing happens when (i) the traffic flow increases and the traffic information is shared using adaptive multi-hop communication and (ii) when the traffic flow remains the same; thus, it is necessary to maintain this information available for the vehicles. The maintenance uses periodical messages and single-hop communication.

The Reactive information sharing is used when a vehicle did not receive sufficient traffic information to find alternative routes due to network issues such as packet collision or sparse topology. In the case to estimate alternative routes, the vehicle verifies if the database contains more than a predefined threshold of amount information and, if necessary, a request-response protocol is executed for all missing information. The alert information sharing protocol is executed when several vehicles execute the request-response protocol, thus the vehicle should react to this information and improve the information availability using periodical messages.

Contribution 3 - Solutions for Route Decision. The Route Decision supports the rerouting process, i.e., the decision-making algorithm that calculates a new lower-cost route considering the local database of traffic information. An important question is: When should decision-making happen? We investigate a Real-time and Periodical decision to find alternatives routes. Real-time decisions demand a high information availability, and they have a better performance dealing with unexpected traffic jams (ex: caused by accidents). Considering Periodical decisions, the vehicles estimate alternative lower-cost 


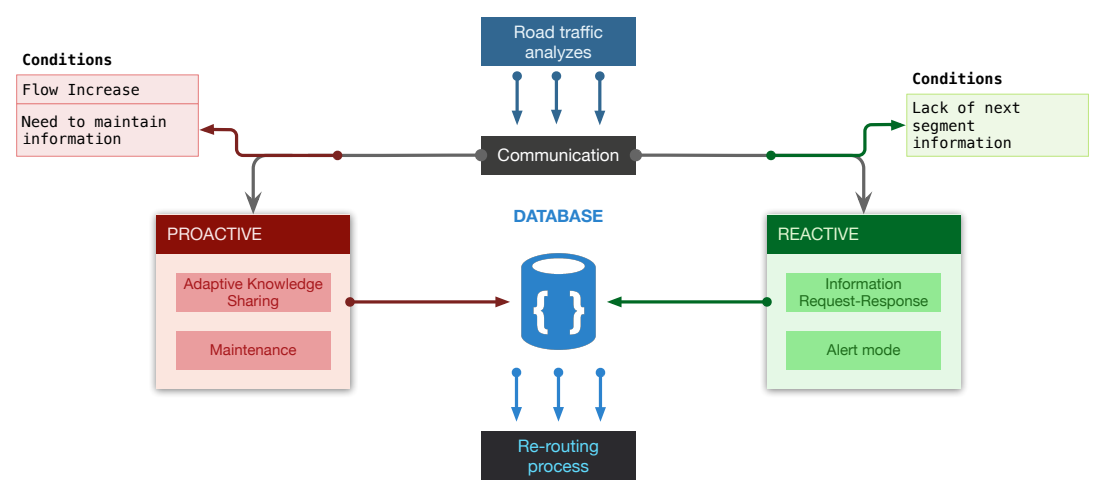

Figure 4. Overview

routes in a predefined time interval, such as one or two minutes. Periodical decisions, instead, do not demand a high use of the network but decrease the response time in decision making. We evaluate both strategies, and since the traffic management system is one of many services executed in the vehicular environment, we adopt periodical decisions for general purpose traffic jams.

\section{Related Work}

Recently, many authors have been addressing the development of ITSs for traffic management using VANETs [Pan et al. 2017, de Souza et al. 2017, Brennand et al. 2019, Wang et al. 2016]. These approaches commonly rely on four communication architectures: Internet-based LTE/5G (Centralized), Vehicle-to-vehicle (V2V) (Distributed), Vehicle-to-infrastructure (V2I) (Decentralized), and Hybrid (V2V + V2I). We briefly review the literature solutions similar to the proposed solutions, as follows.

Centralized architecture [Pan et al. 2017, Wang et al. 2016] use two entities to perform the communication: vehicles and Traffic Management Centers (TMCs) running on a Cloud. Vehicles and TMCs exchange data through the Internet powered by the LongTerm Evolution (LTE) and/or 5G. s-NRR [Wang et al. 2016] is a TMS designed to assist drivers in dealing with the traffic jams caused by traffic events. s-NRR uses induction loops and cameras in the detection of traffic events and notify the TMC. In the presence of an event, the TMC contacts the affected vehicles suggesting alternative routes. Vehicles take their new routes that minimize the travel time. DIVERT [Pan et al. 2017] consists of a centralized system, which uses road segment travel times to calculate new routes. The TMC periodically requests the travel time of the road segments from the vehicles. The server can analyze the global traffic flow and send faster new routes to the vehicles. The central server receives all the data through $4 \mathrm{G}$ communication.

Distributed architectures [de Souza et al. 2017] use 802.11p protocol to add wireless access in vehicular environments (WAVE), where vehicles are the only network entities. PANDORA [de Souza et al. 2017] relies on an opportunistic knowledge dissemination technique, which provides traffic information based on geographical location. During the routes, vehicles analyze their displacement. In predefined turns, they use opportunistic knowledge dissemination to share their traffic views. With the received knowledge, vehicles can perform route calculations. Decentralized architectures, however, use 802.11p to establish the communication between vehicles and Cloudlets/Roadside Units. 
FOX [Brennand et al. 2019] is an example of a decentralized TMS where Cloudlets are FOG entities that monitor the traffic conditions and calculate routes for the vehicles. Vehicles periodically report their traffic view to the Cloudlets. Cloudlets analyze the received data to detect existing/emerging traffic jams. Periodically, vehicles receive a new route from the Cloudlets.

The proposal work differs from the previous works by acting in a distributed manner, reducing the wireless network load, and guaranteeing traffic information sharing among vehicles. By analyzing traffic data in real-time, our proposal proactively and reactively shares the traffic information to provide information for decision-making as soon as the traffic jam starts or maintains the available information.

\section{Results}

We evaluated the algorithms considering a 95\% confidence interval obtained from 33 simulations using a realistic map of the downtown region of Los Angeles city, USA $\left(25 \mathrm{~km}^{2}\right)$. We run all the algorithms under the same set of simulators and parameters, where OMNET++ 5.1.1 and Veins 4.6 run the network simulation over the IEEE 802.11p communication protocol, and SUMO 0.25 manages and performs vehicular mobility. The set of assessed metrics included: (i) Travel Time - the average time for all vehicles to finish their trip; (ii) Distance - distance traveled for all vehicles on their displacements. TMSs increase the distance for vehicles to travel on roads with lower congestion. (iii) Packet Delivery Ratio - the ratio of the number of packets delivered in total to the total number of packets sent.

The main objective of any TMS is to reduce the travel time for all vehicles. The travel time is illustrated in Figure 5(a) considering the different densities of vehicles in the scenario. Considering a low density of vehicles $\left(50\right.$ vehicles $\left./ \mathrm{km}^{2}\right)$, i.e., no congestion, all solutions have similar results. As we increase the number of vehicles and consequently the traffic jams, the DIVERT and the proposed TMS present better results than the other solutions. When the density of vehicles is 300 vehicles $/ \mathrm{km}^{2}$ the proposed solution decreases the travel time by $41 \%, 39 \%$ e $31 \%$, and $10 \%$ compared to the FOX, PANDORA, $s$-NRR and DIVERT, respectively. Thus, the use of proposed displacement analysis to classify the traffic information in order to verify alternative routes presents better results compared to the Highway Capacity Manual used by the PANDORA, the number of vehicles used by the FOX, and $s$-NRR and travel time used by DIVERT. The TMS solutions change the vehicle route to reduce travel time and, consequently, increase the traveled distance. The $s$-NRR and DIVERT solutions have the global view of the traffic information and, for small changes in traffic condition, they estimate new-vehicle routes, increasing, even more, the traveled distance. The distributed solutions are able to find alternative routes with a small increase in distance. For instance, considering 350 vehicles $/ \mathrm{km}^{2}$ PANDORA, proposed TMS, DIVERT, and $s$-NRR increase the distance by $3 \%, 15 \%, 19 \%$, and $23 \%$ over the original route. However, even increasing the traveled distance, the proposed solution is able to find better routes with lower travel time.

The Delivery ratio is shown in Figure 5(c) measures the ratio between successful messages sent and received by the system, and we evaluate this metric only for distributed V2V TMS solutions. It is important to point out that packet collisions may decrease the delivery rate, decreasing the current traffic view accuracy and then affecting vehicular 


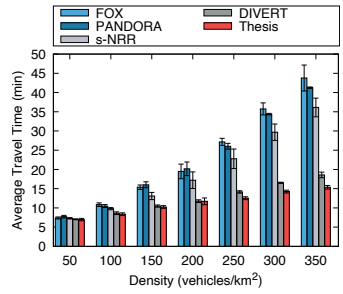

(a) Average Travel Time

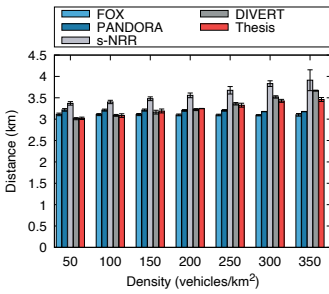

(b) Distance

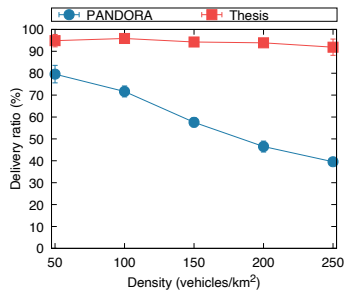

(c) Packet Delivery ratio

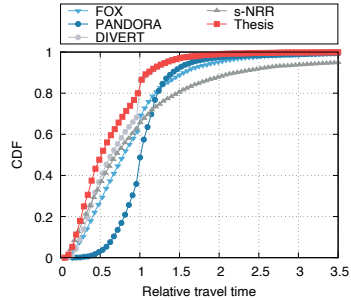

(d) Relative Travel Time

Figure 5. Performance analysis

traffic management. Note that the proposed solution is able to achieve a delivery ratio greater than $90 \%$. Due to the periodical beacon to estimate traffic congestion thus, increasing the packet collisions, PANDORA presents a packet delivery of $40 \%$ when the scenario has a high number of vehicles. Figure $5(\mathrm{~d})$ evaluates the ratio between each TMS solution and the original route (OR) without re-routing considering a Cumulative Distribution Function of the travel time and for 300 vehicles $/ \mathrm{km}^{2}$. The $\mathrm{x}$-axis represents the ratio between the solutions (FOX/OR PANDORA/OR, DIVERT/OR, $s$-NRR/OR Proposed/OR), and the $y$-axis represent the cumulative percentage of vehicles that satisfy the ratio. It is important to point out that the global view of the centralized solutions (DIVERT and $s$-NRR) does not improve its ability to decrease the travel time compared to the proposed solution, which is totally distributed and has a local view of the traffic condition. For instance, the proposed solution, DIVERT, $s$-NRR and PANDORA decrease the travel time compared to the OR in $83 \%, 75 \%, 68 \%$, and $50 \%$, respectively (relative travel time $<1$ ). It is important to point out that all solutions increase the travel time for a group of vehicles compared to the original route; however, the TMS decreases the average global travel time.

This thesis work consisted of an evolutionary process where all results were reported in the following Conferences papers. Each publication introduced a new concept, model, algorithm, or data communication protocol.

- Gomides and Guidoni 2018. WTG workshop - SBRC conference. Protocolo de Roteamento de Veículos fundamentado na Taxa de Ocupação da Via para Redes Veiculares. (Honourable Mention)

- Gomides et al. 2019. SBRC conference (Qualis A4). SGTD: Sistema de Gerenciamento de Tráfego Distribuído para Redes Veiculares.

- Gomides et al. 2019. IEEE DCOSS conference (Qualis A2). FIRE-NRD: A Fully-Distributed and VANETs-based Traffic Management System for Next Road Decision.

- Gomides 2019. LANCOMM (Qualis B4). FAST: Fully-distributed and Vanetbased Traffic Management Architectures.

- Gomides et al. 2020. IEEE DCOSS conference (Qualis A2). RIDER: Proactive and Reactive Approach for Urban Traffic Management in Vehicular Networks.

- Gomides et al. 2020. IEEE SMC conference (Qualis A2). A Traffic Management System to Minimize Vehicle Congestion in Smart Cities.

- Gomides et al. 2020. SBRC conference (Qualis A4). Um Sistema Adaptativo e Colaborativo para Minimizar Congestionamentos utilizando Comunicação entre Veículos. 
The proposed work was also published in ELSEVIER Computer Communications Journal (Qualis A2). Gomides et al. 2020. An Adaptive and Distributed Traffic Management System for Vehicular ad-hoc Networks.

\section{Final remarks}

This thesis's work contributes to the vehicular traffic management system field by proposing solutions that act in a distributed manner, capable of reducing the wireless network load and guaranteeing the share of traffic information among vehicles. By analyzing traffic data in real-time, our proposed approaches proactively share the traffic view to provide information for decision-making as soon as the traffic jam starts. Using a communication protocol, vehicles can request and reactively share their traffic view, anticipating traffic jams. We have conducted an extensive comparative analysis, evaluating our system's performance with recent related approaches through simulations, considering a series of parameters, tools, and scenarios. Over the full development of the thesis, we have compared it with over ten literature solutions such as DIVERT, PANDORA, FOX, s-NRR, among others. The comparison involved assessing more than 30 distinct parameters, contextualizing the urban scenario in five real-world road maps, using several tools and simulators, and executing an uncountable number of simulations. For all considered simulations and parameters, the proposed solutions outperform the literature ones.

\section{References}

[Brennand et al. 2019] Brennand, C. A. R. L., Filho, G. P. R., Maia, G., Cunha, F., Guidoni, D. L., and Villas, L. A. (2019). Towards a fog-enabled intelligent transportation system to reduce traffic jam. Sensors, 19(18).

[Cookson and Pishue 2020] Cookson, G. and Pishue, B. (2020). Inrix global traffic scorecard. INRIX research. Accessed: 2020-12-21.

[de Souza et al. 2017] de Souza, A. M., da Fonseca, N. L. S., and Villas, L. A. (2017). A fully-distributed advanced traffic management system based on opportunistic content sharing. 2017 IEEE International Conference on Communications (ICC), pages 1-6.

[Ekblad 1993] Ekblad, S. (1993). Stressful environments and their effects on quality ot life in third world cities. Environment and Urbanization, 5(2):125-134.

[Madlener and Sunak 2011] Madlener, R. and Sunak, Y. (2011). Impacts of urbanization on urban structures and energy demand: What can we learn for urban energy planning and urbanization management? Sustainable Cities and Society, 1(1):45-53.

[Pan et al. 2017] Pan, J., Popa, I. S., and Borcea, C. (2017). Divert: A distributed vehicular traffic re-routing system for congestion avoidance. IEEE Transactions on Mobile Computing, 16(1):58-72.

[Wang et al. 2016] Wang, S., Djahel, S., Zhang, Z., and Mcmanis, J. (2016). Next road rerouting: A multiagent system for mitigating unexpected urban traffic congestion. IEEE Transactions on Intelligent Transportation Systems, 17:1-12.

[Zhou et al. 2020] Zhou, H., Xu, W., Chen, J., and Wang, W. (2020). Evolutionary v2x technologies toward the internet of vehicles: Challenges and opportunities. Proceedings of the IEEE. 